\title{
The optimal trade credit term and production lot sizes considering shortage cost in manufacturing industry
}

\author{
Yang Guang ${ }^{1}, 2$ a \\ 1.School of International Business Administration, Shanghai University of Finance\& Economics, \\ Shanghai China
}

2.Business School, Jiangsu college of information technology, Wuxi China

aspring1314661@163.com

Keywords: Trade credit, Production lot size, Manufacturing industry

Abstract. As supply chain finance developed, more and more enterprises in manufacturing industry can have better payment terms with their cooperator. This paper consider the decision of the optimal trade credit term and production lot sizes considering shortage cost in manufacturing industry. We build a model with which we can prove that there exist the optimal trade credit term and production lot sizes for enterprises in manufacturing industry.

\section{Introduction}

The manufacturing industry accounts for a great share of the industrial sector in the world especially in developing countries such as China, India. As the manufacturing industry developing, it is encountering a lot of difficulties especially for small and medium sized manufacturing enterprises. Lack of capital is one of them. In other part, as supply chain finance developed, more and more enterprise in manufacturing industry has reconsider its payment strategy with its partner. Trade credit as one hot research area in supply chain finance has been researched by many scholars for a long time. Trade credit can affect the economic order quantity[1], extensive literature has considered the economic order quantities under permissible delay in payment[2,3,4,5,6].In this paper, we derive the seller's optimal trade credit term and lot size policies in an EPQ model. As we know, the longer the trade credit term the higher the opportunity cost and the default risk. In this paper, shortages are allowed to occur. We establish the objective function to find the optimal solution and give some managerial insights.

\section{Notation and assumptions}

The following notation and assumptions are used in the entire paper.

\section{Notation.}

$N$ the seller's trade credit period to his/her buyers in years

$Q$ the seller's production lot size in units

$x$ the average set-up cost per production run in dollars

$c$ the average production cost for making the product per unit in dollars

$p$ the selling price per unit in dollars(with $p>c$ )

$h$ the average stock holding cost per unit per year in dollars

$r$ the seller's annual simple interest rate on opportunity cost

$f$ the unit urgent purchasing price under the condition of shortage

$D(N)$ the annual demand rate in units as a function of the trade credit period $N$

$M$ the annual production rate in units(with $M<D(N)$ )

$\Pi(\mathrm{N}, \mathrm{Q})$ the seller's profit function per year in dollars

$N^{*}$ the seller's optimal trade credit period in years

$Q^{*}$ the seller's optimal production lot size in units

$\Pi^{*}$ the seller's optimal profit per year in dollars 


\section{Assumptions.}

Next, the following assumptions are made to establish the mathematical model.

(1) We assume that the demand rate $D(N)$ is a positive exponential function of the credit period $N$ as $D(N)=K e^{a N}$ where $\mathrm{K}$ and $a$ are positive constants[7].

(2) We assume that the rate of default risk giving the trade credit term $N$ is assumed here to be $F(N)=1-e^{-b N}$ where $b$ is the coefficient of the default risk, which is a positive constant.

(3) The seller offers the buyer a trade credit term of $N$. Since the seller's annual simple interest rate is $\mathrm{r}$, the future value of $\$ 1.00$ received by the seller at time $N$ is equivalent to the present value of $\frac{1}{1+r N}$ received at time 0. Hence, the seller's prese
and opportunity cost is:
$\quad P D(N)[1-F(N)] \frac{1}{1+r N}=P K^{e^{(a-b) N}} / 1+r N$.

\section{Mathematical model and optimal solution}

Based on the above assumptions, the decision model considered here is as follows. The seller must decide its trade Credit term $\mathrm{N}$ and production lot size $Q$ of a single product Simultaneously in order to maximize its profit per year. From the above assumptions and arguments, the annual profit can be expressed as $\Pi(N, Q)=$ present revenue - production cost - set-up cost - holding cost-shortage cost $=$

$$
\begin{aligned}
& P D(N)[1-F(N)] \frac{1}{1+r N}-c M-\frac{M}{Q} x-\frac{Q}{2} h-[D(N)-M] f . \\
= & P K e^{(a-b) N} / 1+r N-c M-\frac{M}{Q} x-\frac{Q}{2} h-\left(K e^{a N}-M\right) f .
\end{aligned}
$$

Then we discuss the seller's optimal solution to production lot size first, and then trade credit term.

\section{Optimal production lot size}

To maximize the annual profit $\Pi(N, Q)$ with respect to $Q$ is equivalent to minimize the annual total cost of the set-up cost and the holding cost, which is

$$
\begin{gathered}
T C(Q)=\frac{M}{Q} x+\frac{Q}{2} h, \frac{\partial T C(Q)}{\partial Q}=0 \\
Q^{*}=\sqrt{\frac{2 M x}{h}} .
\end{gathered}
$$

then we know that the seller's optimal production lot size is

And the minimum total cost of the set-up cost and the holding cost is

$$
T C\left(Q^{*}\right)=\sqrt{2 M x h} \text {. }
$$

Consequently, the seller's decision problem is reduced to a single decision variable $\mathrm{N}$

$$
\Pi(N)=\Pi\left(N, Q^{*}\right)=P K^{e^{(a-b) N}} / 1+r N-c M-\left(K e^{a N}-M\right) f-\sqrt{2 M x h} .
$$

Next, we try to obtain the optimal trade credit term for the seller.

\section{Optimal trade credit term}

In order to find the optimal solution $N^{*}$ of $\Pi(N)$, we derive the first-order necessary condition for $\Pi(N)$ in to be maximized as

$$
\begin{aligned}
& \frac{d \Pi(N)}{d N}=P K\left[\left(e^{(a-b) N}(a-b)(1+r N)-e^{(a-b) N} r\right) /(1+r N)^{2}\right]-K f a e^{a N} \\
& =P K\left[\left(e^{(a-b) N}((a-b)(1+r N)-r) /(1+r N)^{2}\right]-K f a e^{a N}=0\right.
\end{aligned}
$$


From the above equation we can obtain the following results:

Theorem 1. The seller's optimal trade credit term is zero (i.e., $\left.N^{*}=0\right)$ if (1) $(a-b)(1+r N) \leq r$, or (2)

$$
P K\left[\left(e^{(a-b) N}(a-b)(1+r N)-e^{(a-b) N} r\right) /(1+r N)^{2}\right]<K f a e^{a N}
$$

Proof. If $(a-b)(1+r N) \leq r$, then we know from that $\frac{d \Pi(N)}{d N} \leq 0$. trade credit term is set to zero. Likewise, we can easily prove $N^{*}=0$ if $P K\left[\left(e^{(a-b) N}(a-b)(1+r N)-e^{(a-b) N} r\right) /(1+r N)^{2}\right]<K f a e^{a N}$. This completes the proof.

In practice, If $(a-b)(1+r N) \leq r$, then the higher the trade credit term, the lower the present revenue considering default risk and opportunity cost. In this case, the seller should not offer a late payment to its buyer. Similarly, If the marginal revenue increase (i.e., $\left.P K\left[\left(e^{(a-b) N}(a-b)(1+r N)-e^{(a-b) N} r\right) /(1+r N)^{2}\right]\right)$ is less than or equal to the marginal cost increase (i.e., $K f a e^{a N}$ ), then it is no worth of offering a trade credit term to the buyer.

Now we discuss the other condition in which $P K\left[\left(e^{(a-b) N}(a-b)(1+r N)-e^{(a-b) N} r\right) /(1+r N)^{2}\right]=K f a e^{a N}$. Which implies that the seller's optimal trade credit term is

$$
N^{*}=-\frac{1}{b} \ln \left(a f(1+r N)^{2} /[(a-b)(1+r N)-r] P\right)
$$

$>0$.

Notice that the right-hand side of the above equation is also a function of $N$. hence, it's not a closed-form solution.

For the second-order sufficient condition, taking the derivative of with respect to $N$, and re-arranging terms, we get

$$
\begin{aligned}
& \frac{d^{2} \Pi(N)}{d^{2} N}=P K\left[\left(e^{(a-b) N}(a-b)[(a-b)(1+r N)-r]+e^{(a-b) N}(a-b) r-e^{(a-b) N}(1+r N)-e^{(a-b) N}[(a-b)(1+r N)-r] 2(1+r N) r\right) /(1+r N)^{4}\right]-K f a^{2} e^{a N} \\
= & \left.P K e^{(a-b) N}[(a-b)[(a-b)(1+r N)-r]+(a-b) r-2 r(1+r N)[(a-b)(1+r N)-r]) /(1+r N)^{4}\right]-K f a^{2} e^{a N} \\
= & \left.P K e^{(a-b) N}\left[(a-b)^{2}(1+r N)-2 r(1+r N)[(a-b)(1+r N)-r]\right) /(1+r N)^{4}\right]-K f a^{2} e^{a N}
\end{aligned}
$$

Consequently, if $(a-b)^{2}(1+r N) \leq 2 r(1+r N)[(a-b)(1+r N)-r]$, then we know that $\frac{d^{2} \Pi(N)}{d^{2} N}<0$, and hence $\Pi(N)$ is a strictly concave function of $N$.

From the above equations, we can obtain the following theoretical results.

Theorem 2.

(1) If $(a-b)(1+r N)>r$, and $(a-b)^{2}(1+r N) \leq 2 r(1+r N)[(a-b)(1+r N)-r]$, then $\Pi(N)$ has a unique optimal solution $N^{*}>0$

(2) If $(a-b)(1+r N) \leq r$, and $(a-b)^{2}(1+r N)>2 r(1+r N)[(a-b)(1+r N)-r]$, then $\Pi(N)$ has a unique solution $N^{*}=0$

Then under the condition $(a-b)(1+r N)>r$, and $(a-b)^{2}(1+r N) \leq 2 r(1+r N)[(a-b)(1+r N)-r]$, we have $\frac{\partial^{2} \Pi(N, Q)}{\partial N^{2}}<0 \frac{\partial^{2} \Pi(N, Q)}{\partial Q^{2}}=\frac{-2 M x}{Q^{4}}<0$ and $\frac{\partial^{2} \Pi(N, Q)}{\partial Q \partial N}=\frac{x}{Q^{2}}$ So we have $\frac{\partial^{2} \Pi(N, Q)}{\partial N^{2}} \frac{\partial^{2} \Pi(N, Q)}{\partial Q^{2}}-\left[\frac{\partial^{2} \Pi(N, Q)}{\partial Q \partial N}\right]^{2}>0$ 
Hence, the Hessian matrix associated with $\Pi(N, Q)$ is negative definite. Applying Theorem 2, we know that the unique solution $\left(N^{*}, Q^{*}\right)$ is the global maximum solution.

\section{Numerical examples}

In this section, we provide an numerical example to illustrate the theoretical results above.

Example. Let $a=0.3, b=0.2, r=0.05, p=20, f=5, x=5, c=2, h=\$ 1$ per unit per year, $M=10000$ units per year, $K=1000$ units. We first check the condition $(a-b)(1+r N)=0.1+0.005 \mathrm{~N}>r=0.05$

Then we substitute the values of the parameters into (9), and use computer software Maple 18.0 then we obtain a trade credit period $N^{*}=0.2053, Q^{*}=316$. Substituting $N^{*}=0.2053$ and $Q^{*}=316$ into (7), we obtain the optimal annual profit for the seller $\Pi^{*}=14382.6$

\section{Summary}

Manufacturing industry is almost the most important industry for many countries in the world. While many enterprises in this industry lack of development capital especially for SME. This paper try to elaborate that enterprises can by advantage of supply chain fiance to have better payment terms with their parterner. We build a model with which we can prove that there exist the optimal trade credit term and production lot sizes for enterprises.

\section{References}

[1]William Beranek, Financial implications of lot-size inventory models, management science, 13(1967)401-408

[2]Hark Hwang, Seong Whan Shinn, Retailer's pricing and lot sizing policy for exponentially deterioration products under the condition of permissible delay in payments, computers Ops Res, 24(1997)539-547

[3]J-T Teng, On the economic order quantity under conditions of permissible delay in payments, Journal of the operational research society, 53(2002)915-918

[4]Jinn-Tsair Teng, Iris-Pandora Krommyda, A comprehensive extension of optimal ordering policy for stock-dependent demand under progressive payment scheme, European Journal of Operational Research,215(2011)97-104

[5]Chia-Hsien Su, Optimal replenishment policy for an integrated inventory system with defective items and allowable shortage under trade credit, Int.J.Production Economics 139(2012)247-256

[6]Giannetti,et al. What you sell is what you lend? Explaining trade credit contracts, The Review of Financial Studies 24(2013),1261-1298

[7] Jinn-Tsair Teng, Kuo-Ren Lou. Optimal trade credit and lot size policies in economic production quantity models with learning curve production costs, Int.J.Production Economics 155(2014)318-323 